\title{
CARACTERÍSTICAS DE LAS CONCENTRACIONES ELEVADAS DE YODURIA EN ESCOLARES Y ADOLESCENTES DE COLOMBIA
}

\author{
Marisol Galindo(1),a, Ana Yibby Forero(11,b, Jhon Romero(101,c \\ 1 Grupo de Nutrición, Dirección de Investigación en Salud Pública, Subdirección de Investigación Científica y Tecnológica, \\ Instituto Nacional de Salud, Bogotá, Colombia. \\ a Bacterióloga, magíster Salud Pública; ${ }^{\mathrm{b}}$ bacterióloga, magíster en ciencias; ${ }^{\mathrm{c}}$ matemático magíster en Estadística. \\ * Parte de los datos presentados en el presente artículo fueron publicados en el libro de la Encuesta Nacional de la Situación \\ Nutricional (ENSIN) del 2015.
}

\section{RESUMEN}

Objetivos. Determinar las características de las concentraciones elevadas de yoduria en escolares y adolescentes de Colombia. Materiales y métodos. Análisis secundario de la Encuesta Nacional de la Situación Nutricional en Colombia del 2015 en participantes de 5 a 17 años que incluyó la medición de yoduria. Los criterios para medir el estado nutricional del yodo se basaron en la mediana de la concentración de yodo urinario en $\mu \mathrm{g} / \mathrm{L}$ definido por la Organización Mundial de la Salud (OMS), donde valores $<100$ son considerados como deficientes, entre 100-199 adecuada, 200-299 arriba de los requerimientos y $>300$ excesiva. Se realizaron además mediciones de razón de probabilidades a través de un análisis de regresión logística. Resultados. La mediana de yoduria nacional para escolares y adolescentes fue 406,8 $\mu \mathrm{g} / \mathrm{L}$ y 410,8 $\mu \mathrm{g} / \mathrm{L}$ respectivamente, mayor en el área urbana $(410,5 \mu \mathrm{g} / \mathrm{L})$; en la región Atlántica se encontró el mayor nivel para escolares $(423,7 \mu \mathrm{g} / \mathrm{L})$ y en la central para adolescentes $(427,7 \mu \mathrm{g} / \mathrm{L})$. El $4,4 \%$ de escolares y el 2,2\% de adolescentes presentaron deficiencia y en más del $75 \%$ de la población, hubo ingesta excesiva de yodo. Los factores asociados a la ingesta excesiva de yodo en escolares fueron edad, etnia, región e índice de riqueza; y para adolescentes el sexo y área geográfica. Conclusiones. Las concentraciones de yoduria en escolares y adolescentes colombianos es superior al adecuado según OMS, la deficiencia es muy baja y se presenta un grave problema de salud pública por ingesta excesiva de yodo en tres cuartas partes de la población.

Palabras claves: Yodo en orina; Deficiencia de yodo; Exceso de yodo; Escolares (Fuente: DeCS BIREME)

\section{CHARACTERISTICS OF THE HIGH IODURIA CONCENTRATIONS IN SCHOOLCHILDREN AND ADOLESCENTS IN COLOMBIA}

\begin{abstract}
Objetives. To determine the characteristics of high ioduria concentrations in schoolchildren and adolescents in Colombia. Materials and methods. Secondary analysis of the 2015 National Survey of the Nutritional Situation in Colombia in participants aged 5 to 17 years, which included the measurement of ioduria. The criteria to measure the nutritional status of iodine were based on the median urinary iodine concentration in $\mu \mathrm{g}$ / L defined by the World Health Organization (WHO), where values $<100$ are considered deficient, between 100-199 adequate, 200-299 above the requirements and $>300$ excessive. Odds ratio measurements were also performed through logistic regression analysis. Results. The national median iodine for schoolchildren and adolescents was $406.8 \mu \mathrm{g} / \mathrm{L}$ and $410.8 \mu \mathrm{g} / \mathrm{L}$ respectively, higher in the urban area $(410.5 \mu \mathrm{g} / \mathrm{L})$, in the Atlantic region the highest level was found for schoolchildren $(423.7 \mu \mathrm{g} / \mathrm{L})$ and in the central for adolescents $(427.7 \mu \mathrm{g} / \mathrm{L}) .4 .4 \%$ of schoolchildren and $2.2 \%$ of adolescents presented deficiency and in more than $75 \%$ of the population, there was excessive intake of iodine. The factors associated with excessive iodine intake in schoolchildren were age, ethnicity, region and wealth index, and for adolescents gender and geographic area. Conclusions. The iodine concentrations in Colombian schoolchildren and adolescents are higher than adequate according to WHO, the deficiency is very low and a serious public health problem occurs due to excessive iodine intake in three-quarters of the population.
\end{abstract}

Keywords: Iodine in urine; Iodine deficiency; Excess iodine; Schoolchildren (Source: MeSH NLM).

\section{INTRODUCCIÓN}

El yodo - un oligoelemento presente en el suelo, el agua y el aire- es un micronutriente esencial para los humanos. En el cuerpo humano, su función principal es regular la producción de hormo- 
nas tiroideas necesarias para el crecimiento y desarrollo celular, especialmente las células nerviosas ${ }^{(1)}$.

La ingesta deficiente de yodo conduce a presentar manifestaciones clínicas denominadas desórdenes por deficiencia de yodo (DDY). La Organización Mundial de la Salud (OMS) y el Fondo de las Naciones Unidas para la Infancia (UNICEF) recomiendan la yodación universal de la sal como una estrategia segura, rentable y sostenible para garantizar el consumo suficiente de yodo por la población (2).

En los últimos años se han producido grandes avances en la lucha para la eliminación del déficit de yodo. En 1993, la OMS calculaba que a nivel mundial los DDY afectaban a 110 países, mientras que estudios más recientes estiman en 30 el número de países con deficiencia de yodo, ubicados principalmente en Europa y África, países de Latino América por el contrario presentan riesgo de ingesta excesiva de yodo ${ }^{(3)}$.

En Colombia, desde 1947 se establece por ley la yodación de la sal para consumo humano y el 29 de abril de 1988 se llevó a cabo el acto de declaratoria de Colombia como país «Libre de DDY» ${ }^{(4)}$; sin embargo, cada vez es más frecuente encontrar medianas de yodo por encima de los niveles necesarios, según lo recomendado por la OMS, lo cual se convierte en un nuevo problema de salud pública. Esta misma entidad refiere que la población objetivo de la vigilancia por DDY lo constituyen los escolares, las gestantes y los menores de dos años. Los escolares entre 5 a 17 años son considerados una población vulnerable, al respecto se ha reportado una mayor prevalencia de bocio en niños cuyas edades fluctúan entre 8 a 10 años ${ }^{(5-6)}$.

Ingerir dosis elevadas de yodo se asocia con hipotiroidismo en pacientes susceptibles, incluidos aquellos con enfermedad tiroidea autoinmune, tiroiditis subaguda, tiroiditis posparto, hipertiroidismo en pacientes susceptibles con factores de riesgo que incluye bocio nodular no tóxico o difuso y enfermedad de Graves latente ${ }^{(7)}$. En Colombia se han realizado estudios puntuales en lugares como Popayán $(n=139)$ en donde destaca una mediana en la yoduria de $510,3 \mu \mathrm{g} / \mathrm{L}^{(8)}$ y en Quindío $(\mathrm{n}=444)$ en donde se mostró severas deficiencias (zonas rurales) y excesiva ingesta de yodo (población urbana) ${ }^{(9)}$. En el 2002 se realizó un estudio centinela en el que se mostró que el $85 \%$ de la población nacional tenía una ingesta excesiva de yodo ${ }^{(1)}$ y recientemente en la Encuesta de la Situación Nutricional de Colombia 2015, se actualizó la información de la situación de nutrición del yodo en la población colombiana y se reiteró la situación de exceso en población escolar y adolescente colombiana.

El presente estudio tiene como objetivo describir las características de las concentraciones elevadas de yodo en escolares y adolescentes de Colombia.

\section{MATERIALES Y MÉTODOS}

\section{Diseño y población de estudio}

La muestra utilizada de escolares y adolescentes corresponde a información que se recolectó en el componente de vi-

\section{MENSAJES CLAVE}

Motivación para realizar el estudio: Desde hace dos décadas y hasta la Encuesta Nacional de la Situación Nutricional en Colombia del 2015 no se habían realizado estudios poblaciones que dieran cuenta las concentraciones de yoduria en escolares $\mathrm{y}$ adolescentes.

Principales hallazgos: Se evidencia que menos del 5\% presenta deficiencia y más del $75 \%$ ingesta excesiva de yodo y que se asocia con algunas características sociodemográficos.

Implicancias: Se recomienda revisar la norma de fortificación de la sal para disminuir los niveles de yoduria a los sugeridos por OMS.

taminas y minerales de la Encuesta Nacional de la Situación Nutricional (ENSIN) realizada el 2015, una encuesta de hogares, de corte transversal con un diseño de muestra probabilístico, estratificado en varias etapas por conglomerados, conformada por 238 unidades primarias de muestreo y 295 municipios. Se llevó a cabo en 44000 hogares; la muestra de la población estuvo conformada por 22704 escolares y 14106 adolescentes, de los cuales 16151 escolares y 6388 adolescentes formaron parte de la submuestra para la determinación de yoduria ${ }^{(10)}$.

\section{Determinación de yoduria}

Previo diligenciamiento de consentimiento informado, se entregó un recipiente a los participantes y se solicitó $15 \mathrm{~mL}$ de orina recolectada en cualquier momento del día, posteriormente a esos $15 \mathrm{~mL}$ se agregó 3 gotas de ácido clorhídrico para evitar proliferación bacteriana, las muestras fueron inicialmente refrigeradas y posteriormente congeladas a $-20{ }^{\circ} \mathrm{C}$ hasta su procesamiento en el laboratorio de nutrición del Instituto Nacional de Salud (INS). La determinación de la concentración de yodo en orina se realizó mediante espectrofotometría que emplea el persulfato de amonio, reacción de Sandell-Kolthoff modificado por Pino y colaboradores ${ }^{(11)}$.

Los criterios epidemiológicos para medir el estado nutricional del yodo se basaron en la mediana de la concentración de yodo urinario en $\mu \mathrm{g} / \mathrm{L}$ definido por la OMS, en donde describe que valores $<100$ son considerados como deficientes, entre 100199 nutrición adecuada de yodo, 200-299 arriba de los requerimientos y $>300$ ingesta de yodo excesiva ${ }^{(12)}$.

\section{Variables}

Para el análisis sociodemográfico se analizaron variables de edad, sexo, región, área, índice de riqueza y etnia. Colombia tiene definidas seis regiones las cuales fueron usadas para este estudio: Caribe, Central, Oriental, Amazonía y Orinoquía, Pacífica y Bogotá. Respecto a la etnia se precisaron tres: 
la primera población Afro descendiente que incluye, Negro, Mulato, Afrocolombiano, Afro descendiente y Palanquero de San Basilio o raizales; la segunda indígena y la tercera que corresponde al resto de la población, que en Colombia se denomina «sin pertenencia étnica», indica que no se auto reconoce como ninguno de los anteriores. En cuanto al índice de riqueza que corresponde a una medida que ordena a los hogares según condición socioeconómica, es más una medida estructural (no mide ingresos ni consumo) ${ }^{(10)}$.

\section{Análisis estadístico}

Como la distribución del nivel del yodo presente en la orina no sigue una distribución normal (test de Kolmogorov-Sminov), fue necesario utilizar el test de U de Mann-Whitney, también conocida como el test de Wilcoxon rank-sum para determinar si las diferencias entre las diversas subpoblaciones son estadísticamente significativas ${ }^{(13)}$. El otro indicador analizado fue la proporción de ingesta excesiva de yodo. Para probar la hipótesis nula de igualdad entre las proporciones de dos subpoblaciones (variables sociodemográficas) se utilizó el estadístico de prueba que tiene distribución normal estándar teniendo en cuenta que el tamaño de muestras es suficientemente grande. En todos los casos se consideró un nivel de significancia del 5\%, buscando tener un nivel de precisión de cada estimación ${ }^{(14)}$. La variable de concentración de yoduria es una variable continua y lo que se buscó con la estimación de los percentiles 25, 50 y 75 fue tener valores de referencia para analizar la dispersión de la concentración de yoduria. Los valores se calcularon a partir de la variable ponderada por su correspondiente factor de expansión.

Se construyó un modelo de regresión logística para determinar el aporte de las variables sociodemográficas frente a la probabilidad de tener una ingesta excesiva de yodo. Este ejercicio se desarrolló de manera independiente para el grupo poblacional de escolares y de adolescentes, se obtuvieron valores de razón de probabilidades (OR) con intervalos de confianza al $95 \%$ para cada categoría de las variables independientes. Las categorías de referencias de las variables fueron las que tenían la prevalencia más alta ${ }^{(15)}$. En el modelo ajustado se seleccionaron las variables de edad y sexo, teniendo en cuenta que los escolares y adolescentes a medida que aumenta la edad están más expuestos a alimentos fuente de yodo y a la adición de sal a los alimentos. Igualmente, por sexo los hallazgos en Colombia han mostrado que las mujeres escolares y adolescentes también muestran una tendencia a mejores prácticas alimentarias en comparación con los hombres ${ }^{(10)}$.

\section{Consideraciones éticas}

Se contó con el aval de los comités de ética y de investigaciones del Instituto Nacional de Salud. Se tuvieron en cuenta las Normas Científicas, Técnicas y Administrativas de la Investigación en Salud, para un estudio de riesgo mínimo en seres humanos, establecidos en la resolución 008430 de 1993, del Ministerio de Salud de la República de Colombia, y la nor- matividad para estudios de investigación en salud, establecida en la Declaración de Helsinki en 1964 y sus revisiones.

\section{RESULTADOS}

Se evaluaron 16151 escolares entre los 5 a 12 años, encontrándose que la distribución por sexo fue mayor en hombres $(52,3 \%)$, comparado con las mujeres; la mayoría de los escolares residían en el área urbana (70,1\%), el 54,2\% pertenecían a hogares clasificados en cuartil de riqueza más bajo y un $78,8 \%$ fueron auto reconocidos como sin pertenencia étnica (Tabla 1).

De los 6388 adolescentes entre los 13 a 17 años, 56,7\% eran hombres; el 72,1\% y 25,2\% residían en el área urbana y la región central, respectivamente; el 73,8\% pertenecían a hogares clasificados en los cuartiles de riqueza más bajo y bajo y en su mayoría $(81,5 \%)$ fueron auto reconocidos como sin pertenencia étnica (Tabla 2 ).

Por otro lado se puede observar que las medianas de yoduria para los escolares son diferentes por sexo, siendo mayor en los hombres $(415 \mu \mathrm{g} / \mathrm{L})$. Al comparar por edades simples, los niños y niñas de 8 a 12 años presentaron medianas significativamente más altas que las encontradas en los de 5 a 7 años; en relación con el área geográfica se encontró que la mediana es mayor en el área urbana (410,5 $\mu \mathrm{g} / \mathrm{L})$ comparada con la rural $(\mathrm{p}=0,005)$. En cuanto a las regiones se evidenció que la región Atlántica es la que tiene la mediana más alta $(423,7 \mu \mathrm{g} / \mathrm{L})$ y la región Pacífica la más baja $(381,2 \mu \mathrm{g} / \mathrm{L})(\mathrm{p}=0,001)$. Por índice de riqueza y etnia, los pertenecientes al cuartil más bajo y los indígenas son los que presentaron las medianas más bajas, con $400,6 \mu \mathrm{g} / \mathrm{L}$ y $349,3 \mu \mathrm{g} / \mathrm{L}$ respectivamente, y diferencias estadísticamente significativas $(\mathrm{p}=0,001)$ (Tabla 1$)$.

Con respecto a los adolescentes de 13 a 17 años (Tabla 2 ), las medianas de yoduria entre hombres y mujeres presentaron diferencias estadísticamente significativas $(\mathrm{p}=0,001)$, siendo mayor en los hombres $(426,4 \mu \mathrm{g} / \mathrm{L})$. Por edades simples y área geográfica no se encontraron diferencias significativas. Por regiones, se observó diferencias estadísticas entre las medianas de la región Central $(427,7 \mu \mathrm{g} / \mathrm{L})$ y las otras $(\mathrm{p}=0,001)$. Las medianas más bajas se encontraron en los indígenas $(370 \mu \mathrm{g} / \mathrm{L})$.

Según los resultados de este estudio, el 4,4\% de escolares presentó niveles de yoduria por debajo de $100 \mu \mathrm{g} / \mathrm{L}$, correspondiendo a deficiencia, de estos, el 2,1\% tenía niveles de yoduria entre 50 y $99 \mu \mathrm{g} / \mathrm{L}$ y $2,2 \%$ entre 20 y $49 \mu \mathrm{g} / \mathrm{L}$, correspondiendo a deficiencia leve y moderada, respectivamente. Por otra parte, el 13,0\% de estos niños tenía concentraciones entre 200 y $299 \mu \mathrm{g} / \mathrm{L}$, considerado como riesgo de ingesta excesiva; sin embargo, el mayor porcentaje de escolares $(75,2 \%)$ presentó yoduria superior a $300 \mu \mathrm{g} / \mathrm{L}$, lo que es considerado como ingesta excesiva de yodo.

En el grupo de adolescentes, el 2,8\% presentó yoduria menor de $100 \mu \mathrm{g} / \mathrm{L}$, correspondiendo a deficiencia, de estos, 
Tabla 1. Características socio demográficas y distribución de las medianas de yoduria en población escolar colombiana de 5 a 12 años.

\begin{tabular}{|c|c|c|c|c|c|c|}
\hline Características & $\mathbf{n}$ & Proporción \% & Mediana $(\mu \mathrm{g} / \mathrm{L})$ & Percentil $25(\mu \mathrm{g} / \mathrm{L})$ & Percentil $75(\mu \mathrm{g} / \mathrm{L})$ & Valor de $\mathbf{p}^{\star}$ \\
\hline \multicolumn{7}{|l|}{ Sexo } \\
\hline Hombres & 8441 & 52,3 & 415,0 & 307,0 & 485,8 & \multirow{2}{*}{0,001} \\
\hline Mujeres & 7710 & 47,7 & 394,8 & 292,6 & 470,4 & \\
\hline \multicolumn{7}{|l|}{ Edad en años } \\
\hline 5 & 2134 & 13,2 & 392,5 & 287,7 & 475,0 & \multirow{8}{*}{0,001} \\
\hline 6 & 1886 & 11,7 & 399,0 & 296,2 & 479,7 & \\
\hline 7 & 1990 & 12,3 & 399,3 & 275,8 & 475,0 & \\
\hline 8 & 1999 & 12,4 & 412,2 & 307,5 & 481,3 & \\
\hline 9 & 2084 & 12,9 & 410,9 & 313,4 & 481,2 & \\
\hline 10 & 1944 & 12,0 & 410,3 & 292,8 & 474,8 & \\
\hline 11 & 2103 & 13,0 & 409,5 & 304,9 & 488,9 & \\
\hline 12 & 2011 & 12,5 & 410,0 & 319,0 & 482,0 & \\
\hline \multicolumn{7}{|l|}{ Área } \\
\hline Urbana & 11319 & 70,1 & 410,5 & 311,0 & 482,0 & \multirow{2}{*}{0,005} \\
\hline Rural & 4832 & 29,9 & 393,7 & 272 & 472,1 & \\
\hline \multicolumn{7}{|l|}{ Región } \\
\hline Atlántica & 3529 & 21,9 & 423,7 & 324,9 & 494,0 & \multirow{6}{*}{0,001} \\
\hline Oriental & 2543 & 15,7 & 390,2 & 279,5 & 476,0 & \\
\hline Orinoquía y Amazonía & 2878 & 17,8 & 390,2 & 274,3 & 455,4 & \\
\hline Bogotá & 961 & 6,0 & 400,4 & 309,9 & 471,3 & \\
\hline Central & 3714 & 23,0 & 419,5 & 311,0 & 490,0 & \\
\hline Pacífica & 2526 & 15,6 & 381,2 & 268,6 & 455,2 & \\
\hline \multicolumn{7}{|l|}{ Índice de riqueza } \\
\hline Cuartil más bajo & 8761 & 54,2 & 400,6 & 283,5 & 478,5 & \multirow{4}{*}{0,001} \\
\hline Cuartil bajo & 3748 & 23,2 & 411,3 & 310,8 & 481 & \\
\hline Cuartil medio & 2427 & 15,0 & 405,6 & 318 & 477,5 & \\
\hline Cuartil alto & 1215 & 7,5 & 412,0 & 316,3 & 481,3 & \\
\hline \multicolumn{7}{|l|}{ Etnia } \\
\hline Afro descendiente & 1655 & 10,2 & 402,9 & 284,4 & 474,8 & \multirow{4}{*}{0,001} \\
\hline Indígena & 1630 & 10,1 & 349,3 & 174,0 & 450,9 & \\
\hline Sin pertenencia & 12722 & 78,8 & 409,4 & 308,0 & 481,3 & \\
\hline Total & 16151 & 100 & 406,8 & 301,0 & 479,2 & \\
\hline
\end{tabular}

^Prueba Test U Mann Whitney

el 1,2\% tenía niveles de yoduria entre 50 y $99 \mu \mathrm{g} / \mathrm{L}$ correspondiente a deficiencia leve y el 1,6\% entre 20 y $49 \mu \mathrm{g} / \mathrm{L}$, correspondiendo a deficiencia moderada. El 12,4\% de estos adolescentes tenía concentraciones entre 200 y $299 \mu \mathrm{g} / \mathrm{L}$ y el $78,4 \%$ yoduria superior a $300 \mu \mathrm{g} / \mathrm{L}$, lo que es considerado como riesgo de ingesta excesiva e ingesta excesiva de yodo, respectivamente.

$\mathrm{Al}$ analizar los niños con yoduria superior a $300 \mu \mathrm{g} / \mathrm{L}$, se encontró que tres de cada cuatro escolares tienen esta condición con diferencias estadísticamente significativas por sexo $(p=0,01)$, en donde los niños presentaron tres puntos porcentuales más $(76,5 \%)$ comparados con las niñas. Por edades simples se encontraron diferencias estadísticamente significativas $(\mathrm{p}=0,001)$ entre los niños de 7 años de edad que presentaron la prevalencia más baja (72,2 \%) de yoduria elevada en comparación con los niños de 12 años (79,0\%). Con respecto al área geográfica, la prevalencia es mayor en el área urbana $(77,2 \%)$ que en la rural $(\mathrm{p}=0,001)$. Los escolares pertenecientes al cuartil más bajo presentaron las medianas más bajas (72,0\%), mientras que en los demás cuartiles las prevalencias oscilan entre el 76,5 y el 78,4\%. Se observaron diferencias significativas por regiones entre los escolares con medianas clasificadas como ingesta excesiva de yodo, en la región Atlántica $(79,6 \%)$ y lo encontrado en la Pacífica $(69,3 \%)$ en donde se obtuvo el porcentaje más bajo (69,3\%); entre Bogotá y la región Central no hubo diferencias estadísticamente significativas (Tabla 3).

En los adolescentes de 13 a 17 años, persisten las diferencias significativas por sexo y área geográfica respecto a la ingesta excesiva de yodo; al comparar por edades simples solo se observan diferencias significativas $(p=0,039)$ entre los adolescentes de 14 años, quienes tienen la prevalencia más alta $(81,0 \%)$ y los de 16 años con el menor porcentaje $(75,8 \%)$. Por región no se encontraron diferencias significativas, por índice de riqueza se encontró que el cuartil más bajo presentó la menor prevalencia $(76,5 \%)$ y el cuartil alto la más alta $(81,5 \%)$. Por etnia, los auto reconocidos como 
Tabla 2. Características sociodemográficas y distribución de las medianas de yoduria en población adolescente colombiana de 13 a 17 años.

\begin{tabular}{|c|c|c|c|c|c|c|}
\hline Características & $\mathbf{n}$ & Proporción (\%) & Mediana $(\mu \mathrm{g} / \mathrm{L})$ & Percentil $25(\mu \mathrm{g} / \mathrm{L})$ & Percentil $75(\mu \mathrm{g} / \mathrm{L})$ & Valor de $\mathrm{p}^{*}$ \\
\hline \multicolumn{7}{|l|}{ Sexo } \\
\hline Hombres & 3622 & 56,7 & 426,4 & 337,0 & 492,6 & \multirow{2}{*}{0,001} \\
\hline Mujeres & 2766 & 43,3 & 398,0 & 303,0 & 471,0 & \\
\hline \multicolumn{7}{|l|}{ Edad en años } \\
\hline 13 & 1372 & 21,5 & 399,6 & 310,7 & 484,1 & \multirow{5}{*}{0,355} \\
\hline 14 & 1247 & 19,5 & 419,8 & 333,0 & 487,4 & \\
\hline 15 & 1309 & 20,5 & 412,8 & 329,0 & 484,0 & \\
\hline 16 & 1322 & 20,7 & 415,2 & 304,4 & 484,7 & \\
\hline 17 & 1138 & 17,8 & 403,2 & 321,6 & 480,0 & \\
\hline \multicolumn{7}{|l|}{ Área } \\
\hline Urbana & 4607 & 72,1 & 416,4 & 325,4 & 485,9 & \multirow{2}{*}{0,086} \\
\hline Rural & 1781 & 27,9 & 396,2 & 293,6 & 475,0 & \\
\hline \multicolumn{7}{|l|}{ Región } \\
\hline Atlántica & 1329 & 20,8 & 408,9 & 319,0 & 492,8 & \multirow{6}{*}{0,001} \\
\hline Oriental & 1083 & 17,0 & 413,3 & 322,0 & 478,0 & \\
\hline $\begin{array}{l}\text { Orinoquía y Ama- } \\
\text { zonía }\end{array}$ & 1055 & 16,5 & 404,9 & 306,8 & 466,9 & \\
\hline Bogotá & 411 & 6,4 & 410,7 & 320,5 & 484,1 & \\
\hline Central & 1614 & 25,3 & 427,7 & 330,1 & 496,3 & \\
\hline Pacífica & 896 & 14,0 & 394,9 & 299,4 & 466,0 & \\
\hline \multicolumn{7}{|l|}{ Índice de riqueza } \\
\hline Cuartil más bajo & 3211 & 50,3 & 403,7 & 309,0 & 482,0 & \multirow{4}{*}{0,002} \\
\hline Cuartil bajo & 1502 & 23,5 & 420,8 & 333,1 & 488,8 & \\
\hline Cuartil medio & 1057 & 16,5 & 400 & 304,0 & 479,0 & \\
\hline Cuartil alto & 618 & 9,7 & 427 & 336,5 & 484,1 & \\
\hline \multicolumn{7}{|l|}{ Etnia } \\
\hline Afro descendiente & 558 & 8,8 & 411,0 & 316,4 & 489,1 & \multirow{4}{*}{0,001} \\
\hline Indígena & 567 & 9,0 & 370,0 & 258,3 & 457,4 & \\
\hline Sin pertenencia & 5210 & 82,2 & 412,3 & 320,5 & 484,1 & \\
\hline Total & 6388 & 100 & 410,8 & 318,0 & 484 & \\
\hline
\end{tabular}

${ }^{\star}$ Prueba U Mann Whitney

sin pertenecía étnica presentaron la prevalencia más alta $(79,1 \%)$ en comparación con la encontrada en los indígenas que fue $66,5 \%(\mathrm{p}=0,05)$ (Tabla 4$)$.

Los resultados de la aplicación del modelo logístico en la población de 5 a 12 años, que se muestra en la Tabla 5, demostraron que las variables que están relacionadas con la ingesta excesiva de yodo fueron la región geográfica, etnia, región y cuartil de riqueza al que pertenecen. Lo que se identifica es que la población ubicada en el área rural y los indígenas tienen una menor probabilidad de tener ingesta excesiva de yodo, mientras que los ubicados en la región Atlántica y pertenecientes al cuartil de riqueza medio y alto, presentan una mayor probabilidad de tener esta condición. Por otro lado, en los adolescentes, las variables significativas relacionadas con medianas mayores a $300 \mu \mathrm{g} / \mathrm{L}$ fueron área geográfica y etnia, donde la población ubicada en el área rural y los indígenas presentaron menor probabilidad de tener ingesta excesiva de yodo (Tabla 6).

\section{DISCUSIÓN}

Es la primera vez en aproximadamente dos décadas que en Colombia se realiza un estudio poblacional con representatividad nacional y discriminación regional donde se miden los niveles de yodo en orina en población escolar y adolescente. La yoduria es el indicador estándar definido por la OMS para monitorear el estado de yodo, teniendo en cuenta que más del $90 \%$ del yodo consumido se excreta por la orina.

El presente estudio demostró que Colombia tiene una prevalencia de deficiencia total de yodo similar a los datos obtenidos en países como Cuba que presentó el 6,4\% (16), situación que no es comparable con estudios realizados en más 50 países de diferentes partes del mundo, tanto con economías marginales, como en regiones industrializadas, en donde la carencia de yodo es el problema de salud pública más predominante ${ }^{(17)}$. La prevalencia de deficiencia que se reporta para Europa (52,0\%), seguida del Mediterráneo 
Tabla 3. Prevalencia de ingesta excesiva de yodo en escolares colombianos de 5 y 12 años de edad

\begin{tabular}{|c|c|c|c|}
\hline Características & $\mathbf{N}^{*}$ & Prevalencia & IC 95\% \\
\hline \multicolumn{4}{|l|}{ Sexo } \\
\hline Hombres & 8441 & 76,5 & $74,9-78,0$ \\
\hline Mujeres & 7710 & 73,8 & $72,1-75,5$ \\
\hline \multicolumn{4}{|l|}{ Edad en años } \\
\hline 5 & 2134 & 72,2 & $68,5-75,6$ \\
\hline 6 & 1886 & 74,6 & $71,5-77,5$ \\
\hline 7 & 1990 & 71,6 & $68,2-74,9$ \\
\hline 8 & 1999 & 76,5 & $73,0-79,6$ \\
\hline 9 & 2084 & 77,7 & $75,0-80,2$ \\
\hline 10 & 1944 & 73,9 & $70,7-76,9$ \\
\hline 11 & 2103 & 75,6 & $72,5-78,4$ \\
\hline 12 & 2011 & 79,0 & $76,5-81,4$ \\
\hline \multicolumn{4}{|l|}{ Área } \\
\hline Urbana & 11319 & 77,2 & $75,8-78,5$ \\
\hline Rural & 4832 & 70,2 & $67,4-72,9$ \\
\hline \multicolumn{4}{|l|}{ Región } \\
\hline Atlántica & 3529 & 79,6 & $77,3-81,7$ \\
\hline Oriental & 2543 & 71,4 & $68,1-74,5$ \\
\hline $\begin{array}{l}\text { Orinoquía y } \\
\text { Amazonía }\end{array}$ & 2878 & 70,2 & $66,5-73,6$ \\
\hline Bogotá & 961 & 76,6 & $72,4-80,3$ \\
\hline Central & 3714 & 77,4 & $75,4-79,2$ \\
\hline Pacífica & 2526 & 69,3 & $66,1-72,4$ \\
\hline \multicolumn{4}{|l|}{ Índice de Riqueza } \\
\hline Cuartil más bajo & 8761 & 72,0 & $69,9-73,9$ \\
\hline Cuartil bajo & 3748 & 76,5 & $74,5-78,4$ \\
\hline Cuartil medio & 2427 & 78,4 & $76,1-80,6$ \\
\hline Cuartil alto & 1215 & 78,1 & $73,9-81,7$ \\
\hline \multicolumn{4}{|l|}{ Etnia } \\
\hline Afro descendiente & 1655 & 72,0 & $67,7-76,0$ \\
\hline Indígena & 1630 & 59,3 & $49,7-68,2$ \\
\hline Sin pertenencia & 12722 & 76,5 & $75,3-77,7$ \\
\hline Total & 16151 & 75,2 & $74,0-76,4$ \\
\hline
\end{tabular}

* Cantidad de registros de la muestra total usada en el cálculo del indicador de prevalencia.

IC 95\%: intervalos de confianza al 95\%

Oriental $(47,2 \%)$ y África (41,5\%), los menores porcentajes en orden descendente fueron para el Sudeste asiático, Pacífico Occidental y las Américas (30,0\%, 21,2\% y 11,0\%), respectivamente ${ }^{(18)}$.

Los hallazgos en Colombia muestran una mediana de yoduria considerada por la OMS como valores de ingesta excesiva de yodo, con resultados muy superiores a los reportados en Venezuela ${ }^{(19)}$, Brasil ${ }^{(20)}$ y Argentina ${ }^{(21)}$, en donde la mediana en yoduria muestra que están dentro de los rangos adecuados.
Tabla 4. Prevalencia de ingesta excesiva de yodo en adolescentes colombianos de 13 a 17 años de edad

\begin{tabular}{|c|c|c|c|}
\hline Características & $\mathbf{N}^{*}$ & Prevalencia & IC 95\% \\
\hline \multicolumn{4}{|l|}{ Sexo } \\
\hline Hombres & 3622 & 81,1 & $79,3-82,9$ \\
\hline Mujeres & 2766 & 75,5 & $72,7-78,0$ \\
\hline \multicolumn{4}{|l|}{ Edad en años } \\
\hline 13 & 1372 & 78,3 & $74,4-81,7$ \\
\hline 14 & 1247 & 81,0 & $77,7-83,8$ \\
\hline 15 & 1309 & 79,2 & $75,6-82,4$ \\
\hline 16 & 1322 & 75,8 & $71,8-79,4$ \\
\hline 17 & 1138 & 78,0 & $74,2-81,4$ \\
\hline \multicolumn{4}{|l|}{ Área } \\
\hline Urbana & 4607 & 79,9 & $78,1-81,7$ \\
\hline Rural & 1781 & 74,1 & $70,6-77,3$ \\
\hline \multicolumn{4}{|l|}{ Región } \\
\hline Atlántica & 1329 & 79,9 & $76,4-83,1$ \\
\hline Oriental & 1083 & 79,0 & $75,2-82,4$ \\
\hline Orinoquía y Amazonía & 1055 & 76,8 & $70,9-81,8$ \\
\hline Bogotá & 411 & 78,8 & $73,6-83,1$ \\
\hline Central & 1614 & 79,1 & $76,0-81,9$ \\
\hline Pacífica & 896 & 74,7 & $70,3-78,7$ \\
\hline \multicolumn{4}{|l|}{ Índice de Riqueza } \\
\hline Cuartil más bajo & 3211 & 76,5 & $74,0-78,8$ \\
\hline Cuartil bajo & 1502 & 80,2 & $77,3-82,8$ \\
\hline Cuartil medio & 1057 & 77,6 & $73,5-81,1$ \\
\hline Cuartil alto & 618 & 81,5 & $76,7-85,4$ \\
\hline \multicolumn{4}{|l|}{ Etnia } \\
\hline Afro descendiente & 558 & 76,7 & $71,0-81,5$ \\
\hline Indígena & 567 & 66,5 & $57,5-74,4$ \\
\hline Sin pertenencia & 5210 & 79,1 & $77,4-80,7$ \\
\hline Total & 6388 & 78,4 & $76,8-80,0$ \\
\hline
\end{tabular}

* Cantidad de registros de la muestra total usada en el cálculo del indicador de prevalencia.

IC 95\%: intervalos de confianza al 95\%

Otras investigaciones, que aun cuando reportan medianas inferiores a las encontradas en este estudio, siguen siendo considerados por la OMS como arriba de los requerimientos, por ejemplo, el estudio del departamento del Quindío (Colombia) con una mediana de yoduria de $272 \mu \mathrm{g} / \mathrm{L}^{(9)}$, Salvador en donde se obtuvieron medianas de $206 \mu \mathrm{g} / \mathrm{L}^{(22)} \mathrm{O}$ estudios en Perú, que mostraron de medianas que van desde $258,5 \mu \mathrm{g} / \mathrm{L}$ a $262,4 \mu \mathrm{g} / \mathrm{L}^{(6-23)}$. Otra es la situación de Costa Rica ${ }^{(24)}$, que presentó una mediana de $314 \mu \mathrm{g} / \mathrm{L}$ que, aun estando clasificada como ingesta excesiva de yodo por la OMS, es inferior a la mediana encontrada en Colombia.

Son pocos los estudios que reportan datos superiores a los encontrados en este estudio y que son clasificados por la OMS como ingesta excesiva de yodo, uno de ellos fue el realizado en 139 escolares de Popayán, ciudad de Colombia, en donde la mediana de yoduria fue de $510,3 \mu \mathrm{g} / \mathrm{L}^{(8)} \mathrm{y}$ los descritos en Paraguay que reportó medianas de $437 \mu \mathrm{g} / \mathrm{L}^{(25)}$. 
Tabla 5. Análisis de factores asociados a la ingesta excesiva de yodo según características sociodemográficas en escolares colombianos de 5 a 12 años

\begin{tabular}{|c|c|c|c|c|}
\hline Variable & OR crudo & IC 95\% & OR ajustado* & IC 95\% \\
\hline Edad & 1,04 & $1,01-1,07$ & 1,04 & $1,01-1,07$ \\
\hline \multicolumn{5}{|l|}{ Sexo } \\
\hline Mujer & 1.00 & & 1,00 & \\
\hline Hombre & 1,16 & $1,03-1,30$ & 1,16 & $1,03-1,30$ \\
\hline \multicolumn{5}{|l|}{ Área } \\
\hline Urbana & 1,00 & & 1,00 & \\
\hline Rural & 0,70 & $0,59-0,82$ & 0,70 & $0,59-0,82$ \\
\hline \multicolumn{5}{|l|}{ Etnia } \\
\hline Afrodescendiente & 1,00 & & 1,00 & \\
\hline Indígena & 0,56 & $0,35-0,92$ & 0,57 & $0,35-0,93$ \\
\hline Sin pertenencia étnica & 1,27 & $1,03-1,56$ & 1,27 & $1,03-1,56$ \\
\hline \multicolumn{5}{|l|}{ Región } \\
\hline Otras regiones & 1,00 & & 1,00 & \\
\hline Atlántica & 1,40 & $1,19-1,63$ & 1,40 & $1,19-1,63$ \\
\hline Central & 1,17 & $1,02-1,33$ & 1,16 & $1,02-1,33$ \\
\hline \multicolumn{5}{|l|}{ Cuartil riqueza } \\
\hline Cuartil más bajo & 1,00 & & 1,00 & \\
\hline Cuartil bajo & 1,26 & $1,10-1,47$ & 1,27 & $0,99-1,35$ \\
\hline Cuartil medio & 1,41 & $1,20-1,67$ & 1,41 & $1,20-1,67$ \\
\hline Cuartil alto & 1,39 & $1,08-1,78$ & 1,38 & $1,08-1,77$ \\
\hline
\end{tabular}

* Ajustado por edad y sexo

OR: Odds ratio, IC 95\%: intervalos de confianza al 95\%

Diferentes estudios señalan que el incremento de las medianas podría tener causas diversas como la adición de altos niveles de yodo a la sal, concentraciones elevadas de yodo en agua potable, consumo de alimentos con altos niveles de yodo u otros factores no estudiados que pudieran estar influyendo en la excesiva excreción de yodo en orina ${ }^{(26)}$.

Diversos autores han definido que la causa más probable para que se presente esta condición en la población colombiana, es la alta concentración de yodo definida en la norma colombiana para la fortificación de la sal con yodo ${ }^{(8-9)}$, que se encuentra entre 50 y $100 \mathrm{mg} / \mathrm{kg}{ }^{(27)}$, considerada a nivel de Latino América como una de las dosis más elevadas, comparadas con otros países como Argentina, Perú, Uruguay y México en donde la concentración de yodo esta entre los 20-40 ppm ${ }^{(28)}$ y muy por encima de la recomendación de la OMS ${ }^{(29)}$. China, por ejemplo, presentó una situación similar a lo que sucede en Colombia y realizó la reducción de la concentración de yodo en sal, logrando una disminución de la yoduria en escolares de 8 a 10 años a tal punto que logró que su mediana pasara de ser clasificada por OMS como niveles por encima de lo recomendado a niveles adecuados ${ }^{(30)}$.

Además de la adición de la sal en Colombia, los datos mostraron que los hombres tanto en población escolar como adolescente son los que presentaron mayores valores de medianas considerados como ingesta excesiva de yodo, lo cual puede estar relacionado con la mayor ingesta alimentaria comparada con mujeres. Frente al análisis por área geográfica, nuestros hallazgos coinciden con lo reportado por otros estudios, donde se encontró que las medianas de yoduria son mayores en el área urbana que en escolares que habitan en áreas rurales ${ }^{(9)}$.

En cuanto a la etnia e índice de riqueza, no es posible hacer comparaciones con otros estudios por ausencia de evidencia de estos análisis, pero de acuerdo a los resultados, hay más escolares y adolescentes con medianas de yoduria consideradas como ingesta excesiva de yodo en la población clasificada en el cuartil alto de riqueza y en el grupo sin pertenecía étnica, esto estaría relacionado con los hábitos de alimentación, acceso a alimentos industrializados o procesados para consumo humano, siendo estos los únicos que están cubiertos con el decreto de yodación de la sal y que tienen aditivos como yodato de calcio y potasio, por lo cual la sal no es la única fuente de yodo ya que el agua potable incluye en el proceso de potabilidad la adición de yodo ${ }^{(27)}$.

Las fortalezas de este estudio son el tamaño y la representatividad de la muestra respecto al territorio nacional, que permitió análisis desagregados por todas las variables definidas en la ENSIN. Además, se resalta la calidad de las muestras de orina, sin embargo, no se realizó evaluación de función y autoinmunidad tiroidea y no se contó con información relacionada a contenido de yodo en la sal de mesa de las personas encuestadas, ni se estimaron los niveles de yodo o consumo de sal en la ingesta. 
Tabla 6. Análisis de factores asociados a la ingesta excesiva de yodo según características sociodemográficas en adolescentes colombianos de 13 a 17 años

\begin{tabular}{|c|c|c|c|c|}
\hline Variable & OR crudo & IC 95\% & OR ajustado* & IC 95\% \\
\hline Edad & 0,97 & $0,91-1,03$ & 0,95 & $0,90-1,03$ \\
\hline \multicolumn{5}{|l|}{ Sexo } \\
\hline Mujer & 1,00 & & 1,00 & \\
\hline Hombre & 1,40 & $1,16-1,68$ & 1,40 & $1,17-1,69$ \\
\hline \multicolumn{5}{|l|}{ Área } \\
\hline Urbana & 1,00 & & 1,00 & \\
\hline Rural & 0,72 & $0,58-0,88$ & 0,71 & $0,58-0,88$ \\
\hline \multicolumn{5}{|l|}{ Etnia } \\
\hline Afrodescendiente & 1,00 & & 1,00 & \\
\hline Indígena & 0,61 & $0,37-0,98$ & 0,61 & $0,38-0,97$ \\
\hline Sin pertenencia étnica & 1,15 & $0,85-1,56$ & 1,16 & $0,85-1,57$ \\
\hline \multicolumn{5}{|l|}{ Región } \\
\hline Otras regiones & 1,00 & & 1,00 & \\
\hline Atlántica & 1,13 & $0,90-1,42$ & 1,13 & $0,89-1,43$ \\
\hline Central & 1,05 & $0,86-1,30$ & 1,06 & $0,86-1,30$ \\
\hline \multicolumn{5}{|l|}{ Cuartil riqueza } \\
\hline Cuartil más bajo & 1,00 & & 1,00 & \\
\hline Cuartil bajo & 1,24 & $1,00-1,53$ & 1,25 & $1,00-1,54$ \\
\hline Cuartil medio & 1,06 & $0,83-1,36$ & 1,07 & $0,83-1,39$ \\
\hline Cuartil alto & 1,35 & $0,98-1,86$ & 1,37 & $0,99-1,88$ \\
\hline
\end{tabular}

${ }^{*}$ Ajustado por edad y sexo

OR: odds ratio, IC 95\%: intervalos de confianza al 95\%

En conclusión, es fundamental revisar el proceso de fortificación para modificar el decreto vigente de yodación de sal y disminuir su concentración a niveles sugeridos por la OMS, además de implementar sistemas de monitoreo de la situación de yoduria en la población para controlar los niveles de ingesta excesiva de yodo. Teniendo en cuenta lo anterior se sugiere hacer investigaciones para evaluar el acceso a fuentes de yodo, hábitos alimentarios asociados al consumo de sal y niveles de yodo en sal de consumo humano.

La deficiencia de yodo en esta población es baja, pero es necesario identificarla para implementar estrategias específicas y disminuir el riesgo de DDY; sin embargo, el problema de salud pública en la población escolar y adolescente en Colombia es la ingesta excesiva de yodo, que además de estar principalmente asociada a la alta concentración de yodo adicionada en el proceso de fortificación de la sal, se demostró que a mayor edad, vivir en el área urbana, estar ubicados

\section{REFERENCIAS BIBLIOGRÁFICAS}

1. Juraci C, Santos IS, Black RE, Chrestani MAD, Duarte FA, Nilson EAF. Iodine Status of Brazilian School-Age Children: A National Cross-Sectional Survey. Nutrients. 2020;12(4):1077. doi: 10.3390/nu12041077.

2. Katagiri R, Yuan X, Kobayashi S, Sasaki. Effect of excess iodine intake on thyroid diseases in different populations: A systematic en la región Atlántica y Central, pertenecer al cuartil alto de riqueza y ser reconocidos como sin pertenencia étnica, aumenta la probabilidad de los escolares a tener ingesta excesiva de yodo; la misma situación se observó para adolescentes hombres y residentes de la zona urbana.

Contribuciones de los autores: MG, AF y JR han participado en la concepción del artículo, enfoque científico del proyecto, procesamiento y análisis de la información, su redacción y aprobación de la versión final. Además, JR realizó la selección de la muestra, marco muestral. AF obtuvo financiamiento del proyecto.

Financiamiento: Agradecemos y damos los créditos a Instituto Nacional de Salud, Ministerio de Salud y protección Social, Universidad Nacional de Colombia, Prosperidad Social e Instituto Colombiano de Bienestar Familiar por el apoyo económico para la realización del proyecto.

Conflicto de interés: Los autores declaran no tener conflictos de interés.

review and meta-analyses including observational studies. PloS ONE, 2017;12(3):e0173722. doi: 10.1371/journal.pone.0173722.

3. Fernández M, Menéndez E, Cadórniga F, Fernández J, Delgado E. Iodine nutritional status in Asturian schoolchildren. Endocrinol Diabetes Nutr. 2017;64(9):491-497. doi: 10.1016/j.endinu.2017.06.003. 
4. Ministerio de Salud, Instituto Nacional de Salud, Fondo de las Naciones Unidas para la Infancia, Instituto Colombiano de Bienestar Familiar, Instituto Nacional de Vigilancia de Medicamentos y alimentos, Organización Panamericana de la Salud, Organización Mundial de Salud. Vigilancia epidemiológica de los desórdenes por deficiencia de yodo: Colombia 1997. Bogotá; 1998. 19 p.

5. Tarqui C, Sánchez J, Alvarez D, Jordán T, Fernández I. Concentraciones de yodo en orina y en sal de consumo en mujeres entre 12 a 49 años del Perú. Rev Peru Med Exp Salud Pública. 2015;32(2):252-8.

6. Tarqui C, Alvarez D, Fernández I. Yoduria y concentración de yodo en sal de consumo en escolares peruanos del nivel primario. Rev Peru Med Exp Salud Pública. 2016;33(4):689-94. doi:10.17843/rpmesp.2016.334.2552.

7. De la Vieja A, Santisteban P. Role of iodide metabolism in phisyology and cancer. Endocr Relat Cancer. 2018;25(4):225-245. doi: 10.1530/ ERC-17-0515.

8. Vargas HU, Bastidas B, Perdomo M, Vargas H. Iodine nutritional status its role in detection of thyroid autoantibodies and possibly, thyroid autoimmunity, in a scholar population declared "free from iodine-deficiency disorders". Medicina. 2015;37(2):122-139.

9. Gallego ML, Loango N, Londoño AL, Landázuri P. Niveles de excreción urinaria de yodo en escolares del Quindío, 2006-2007. Rev Salud Publica. 2009;11(6):952-960.

10. Instituto Colombiano de Bienestar Familiar, Ministerio de Salud y Protección Social, Instituto Nacional de Salud, Departamento Administrativo para la Prosperidad Social. Encuesta Nacional de la Situación Nutricional en Colombia, ENSIN 2015. Bogotá D.C.; 2015. Disponible en: http://www.ensin.gov.co/Documents/Resultadosgenerales-ENSIN-2015-preliminar.pdf.

11. Pino S, Fang S, Braverman LE.Ammonium persulfate: a safe alternative oxidizing reagent for measuring urinary iodine. Clinical Chemistry 1996; 42(2): 239-243.

12. World Health Organization. Salt Reduction and Iodine Fortification Strategies in Public Health. Geneva, 2014. Disponible en http:// apps.who.int/iris/bitstream/10665/101509/1/9789241506694_eng. pdf?ua=1.

13. Conroy R. What hypotheses do "nonparametric" two-group tests actually test. Stata J. 2012;12(2):182-190. doi: 10.1177/1536867X1201200202

14. Díaz G, Morales M. Análisis Estadístico de datos categóricos. Bogotá: Universidad Nacional de Colombia. 2009.

15. Cerda J, Vera C, Rada G, Odds ratio: aspectos teóricos y prácticos. Rev Med Chile. 2013.141(10):1329-1335. doi: 10.4067/S003498872013001000014.

16. Terry B, Zulueta D, De la Paz M, Rodríguez A, Alavez S. Iodine deficiency in Cuba. Rev Cubana Hig Epidemiol. 2013;51(3):242-254.

17. Vargas H, Sierra C, Holguín C, Cristancho L. Trastornos asociados a la deficiencia de Yodo: Vigilancia permanente es deficitaria en zonas vulnerables. Medicina. 2012;34(2): 119-145.
18. Benoist B, McLean E, Andersson M. Iodine deficiency in 2007: Global progress since 2003. Food Nutr Bull. 2008;29(3):195-202. doi: 10.1177/156482650802900305.

19. Caballero L. Yoduria en escolares y embarazadas del estado Trujillo, Venezuela 2007-2008. Rev Argent Endocrinol Metab. 2011;48(4):206-211.

20. Navarro AM, Oliveira LA, Meirelles C, Costa TMB. Lodação do sal e ingestão excessiva de iodo em crianças. Arch Latinoam Nutr. 2010;60(4):355-359.

21. Milani ML, Paéz JM, Parco Parisi MA, Parco Parisi L, Pécora R, Bernatené D, et al. Monitoreo de DDI en la provincia de La Rioja. Rev Argent Endocrinol Metab. 2010;47(1):53-7.

22. Ministerio de Salud de El Salvador. Estudio Nacional de yoduria, evaluación del estado nutricional y de alimentos fortificados en escolares de primero y segundo grado. El Salvador; 2012. 70 p.

23. Miranda M, Chávez H, Aramburú A, Tarqui C. Estado nutricional de yodo en alumnos de escuelas públicas del nivel primario en el Perú, 2009. Lima: Instituto Nacional de Salud; 2011.

24. Ministerio de Salud, República de Costa Rica, Fondo de las Naciones Unidas para la infancia. Yodar la sal una política de salud: la experiencia de Costa Rica. Primera edición. San José de Costa Rica; 2013. 25 p.

25. Jara JA, Pretell E, Jara E, Jara M, Jara J, Jara E. Exceso de yodo en la sal riesgo de hiper e hipotiroidismo - situación del control de la deficiencia de yodo en Paraguay. Tend Med. 2015; 10(10):19-24.

26. Terry B, Zulueta D, De la Paz L, Rodríguez A, Alavez E, Turcios S. La deficiencia de yodo en Cuba. Rev Cubana Hig Epidemiol. 2013;51(3):242-254.

27. Ministerio de Salud Colombia. Decreto 547 1996: Reglamentación del Título V de la Ley 09 de 1979, en cuanto a la expedición del registro Sanitario y a las condiciones sanitarias de producción, empaque y comercialización, al control de la sal para consumo humano y se dictan otras disposiciones sobre la materia. Bogotá; 1996. 10 p.

28. Fondo de las Naciones Unidas para la Infancia, Organización Panamericana de la Salud, Iodine Global Network. Eliminación sostenible de los desórdenes por deficiencia de yodo en Latinoamérica 2014. Disponible en: http://www.ign.org/cm_data/Eliminacion_Sostenible_-_Espaol_Doc.pdf

29. World Health Organization, United Nations Children's Fund, International Council for Control of Iodine Deficiency Disorders. Assessment of iodine deficiency disorders and monitoring their elimination. A guide for programme managers. 3rd ed. WHO/UNICEF/ICCIDD. Geneva: WHO; 2008.98 p.

30. Zou Y, Lou X, Ding G, Mo Z, Zhu W, Mao G. Iodine nutritional status after the implementation of the new iodized salt concentration standard in Zhejiang Province, China. BMC Public Health. 2014;14:836. doi: 10.1186/1471-2458-14-836. 